\title{
Burn-in policies for products having dormant states
}

\author{
Shaomin $\mathrm{Wu}^{1}$, Derek Clements-Croome \\ School of Construction Management and Engineering, The University of Reading, \\ RG6 6AW, Reading, The United Kingdom.
}

\begin{abstract}
Many systems might have a long time dormant period, during which the systems are not operated. For example, most building services products are installed while a building is constructed, but they are not operated until the building is commissioned. Warranty terms for such products may cover the time starting from their installation times to the end of their warranty periods. Prior to the commissioning of the building, the building services products are protected by warranty although they are not operating. Developing optimal burn-in policies for such products is important when warranty cost is analysed. This paper considers two burn-in policies, which incur different burn-in costs, and have different burn-in effects on the products. A special case about the relationship between the failure rates of the products at the dormant state and at the operating state is presented. Numerical examples compare the mean total warranty costs of these two burn-in policies.
\end{abstract}

Key words: burn-in, warranty policy, failure pattern, cost, reliability

\section{Introduction}

Warranty is a duty attached to a product and requires manufacturers to offer pre-specified compensation to buyers when a product fails to perform its design functions under normal usage within the warranty period. Nowadays, product warranty becomes increasingly more important in consumer and commercial transactions, and is widely used and serves many purposes. These purposes include protection for both the manufacturer and the buyer; an indicator of

$\overline{1}$ Current E-mail address: s.m.wu@kent.ac.uk//Suggested citation: Wu, S. and Clements-Croome, D. (2007) Burn-in Policies for Products Having Dormant States. Reliability Engineering and System Safety, 92 (3). pp. 278-285. 
product quality and reliability; a promotional tool to gain reputation; assuring buyers against products which do not perform as promised. Product warranty plays an important role in case there is any dispute resolution between buyer and manufacturer. This in turn poses serious challenges to legislators in terms of formulating sensible warranty policy legislation that will protect both the buyers and manufacturers interests.

Warranty policy is an important factor influencing different stages of the whole lifecycle of products including the development stage(see $[1,2]$ ), and the operation and maintenance stage (e.g., [3]). Warranty is mainly utilised in the manufacturing industries such as the automobile industry (see [4,5]), and the information technology industry (e.g., [6]).

Warranty policies for a product sold are usually developed on the basis of the failure pattern of the product. A typical failure pattern against time is divided into three segments: an infant mortality period, usually marked by a rapidly decreasing failure rate; a random failure period, where the failure rate continues at a steady level; and a period of an increasing failure rate representing the onset of product wear-out.

Different types of failures may occur within the three periods. From a client satisfaction perspective, infant moralities are unacceptable. They can be caused by design deficiencies of the product, poor quality control, poor process control or workmanship during manufacture or installation. To reduce possible damage from early failures, one way is to perform a burn-in procedure, which is carried out by operating the products under electrical or thermal conditions that approximate to the working conditions in field operation.

Building services products are installed in a building to support the functions required. Most building products are protected under a kind of warranty or insurance policy starting from the installation period. These products, for example, heating, ventilation, air conditioning (HVAC) systems and so forth, are installed while a building is constructed. They are not usually operated until the building is completed and commissioned. The time from the installation to the commissioning, is called a dormant state and may take several years. It is not a short time period comparing with their whole life time.

Unlike products that are used immediately after purchase, the building services products have a dormant state and:

- the products may age and deteriorate, and may therefore fail to function when they are put into use at the commissioning time, and

- no inspection, or maintenance is conducted on the building services products during this non-operable period.

As there may be no inspection or maintenance until the building is commis- 
sioned, any failures within the dormant period occur with no cost for maintenance. Burn-in policies for such products can therefore be different from those without any dormant states.

Burn-in is an engineering method that involves testing of items for a given time, and then releasing those items meet service test to the user [7]. The items that fail during the burn-in procedure will be scrapped or repaired; only those that survive the burn-in procedure will be considered to be of good quality. Burn-in could be performed on the basis of a system (see $[8,9]$ ) or an individual component (e.g., $[10,11]$ ). The systems might be electronic systems such as circuit boards or mechanical systems including air conditioners, or subsystems like an aircraft electrical subsystem, and the components would be various types of chips, printed circuits, condensers, fans, and so forth.

A burn-in procedure is usually made in three steps: (1) estimate a lifetime distribution for a given population, (2) assess the suitability of a burn-in procedure based on the lifetime distribution, and (3) select a optimization criterion in order to determine the duration of the burn-in. In the reliability literature, much research focuses on the third step. The burn-in optimization criteria includes performance-based methods [12,13], and cost-based methods $[14,15,16,17]$.

There is considerable research on burn-in policies. For a more comprehensive understanding of different types of burn-in policies, the reader is referred to the work of Blischke [18], Block [19], Jensen[20], and Kuo and Kuo [21].

This paper considers two scenarios for products having dormant states.

- Scenario A - Policy A is performed, which implements only one burn-in procedure under a normal operating environment.

- Scenario B- Policy B is performed, which performs one burn-in procedure under a dormant environment and follows a burn-in procedure under a normal operating state. Costs of the burn-in procedure under the dormant environment is cheaper than under the operating environment, but its burnin effect under the dormant environment is less than that under the normal operating environment.

The paper is structured as follows. Section 2 presents denotations and assumptions on failure rates of the products under both dormant states and normal operating states, warranty policies and costs. Section 3 obtains the mean total warranty costs under the two scenarios. Section 4 gives the mean total warranty costs when a special case about the failure rate is considered, and a numerical example is also presented to compare the warranty costs. Section 5 provides concluding remarks. 


\section{Assumptions and denotations}

\subsection{Assumptions}

The following assumptions are held.

- The product installed in a building is in a dormant state from its installation time 0 to commissioning time $t_{0}$, and put into use at time $t_{0}$. No maintenance on the product is conducted within the time interval $\left(0, t_{0}\right)$. There is an inspection at time $t_{0}$; maintenance will be carried out immediately if any failure is found. Inspection time and maintenance time is assumed to be negligible.

- The product has failure rates as follows

$$
r(t)=\left\{\begin{array}{l}
r_{d}(t) \text { at the dormant state } \\
r_{u}(t) \text { at the operating state }
\end{array}\right.
$$

Assume

$$
r_{u}(t) \text { is }\left\{\begin{array}{l}
\text { strictly decreasing for } 0 \leq t_{u} \leq t_{1} \\
\text { constant } \quad \text { for } t_{1} \leq t_{u} \leq t_{2} \\
\text { strictly increasing for } t_{2} \leq t_{u} \leq \infty
\end{array}\right.
$$

where $t_{u}$ is operating time, $t_{0}<t_{1}$.

- The warranty term starts from the commissioning time. A free replacement non-renewing warranty (FRW) policy is considered in this paper. The warranty covers each product for a warranty period $w$.

- The products are protected by a free-replacement non-renewing warranty (FRW) policy. That is, the buyer is charged no cost on replacement within the warranty period $w$ with $w>t_{0}$. The warranty period $w$ is fixed, and cannot be renewed.

\subsection{Denotation}

Denote the life distribution of the product in the dormant state as $F_{d}(t)=$ $1-e^{-\int_{0}^{t} r_{d}(x) d x}$, and the life distribution of the product in the normal operating state as $F_{u}(t)=1-e^{-\int_{0}^{t} r_{u}(x) d x}, \bar{F}_{u}(t)=1-F_{u}(t)$ and $\bar{F}_{d}(t)=1-F_{d}(t)$.

For costs there are several additive components as follows: 
$c_{0}$ : manufacturing cost per product without burn-in;

$c_{1 d}$ : fixed setup cost of burn-in per product under a dormant environment;

$c_{1 u}$ : fixed setup cost of burn-in per product under an normal operating environment;

$c_{2 d}$ : cost per product time of burn-in per product under a dormant environment;

$c_{2 u}$ : cost per product time of burn-in per product under an normal operating environment;

$c_{3}$ : shop replacement cost per failure;

$c_{4}$ : extra replacement cost per failure during the warranty period.

\section{Main results}

The failure rate of a product with a dormant state is different from those without a dormant states. It can be lower within the dormant period than that within the normal operating period; and it is not a continuous function over the whole life cycle. Figure 1 shows a particular scenario, where the Xaxis is time and the $\mathrm{Y}$-axis is the failure rate. We call this bathtub curve a broken bathtub curve.

As the broken bathtub curve is different from the normal one, the burn-in policy should be developed accordingly. Policy A and B are defined as:

- Policy A: a burn-in procedure is carried out for $b_{1 u}$ time units under a normal operating environment.

- Policy B: a burn-in procedure is divided into two-stages. The first stage is carried out for $b_{d}$ time units under a dormant environment, and then for the second stage $b_{2 u}$ time units under a normal operating environment.

Denote the cost for policy A as $C_{1}\left(b_{1 u}\right)$, and the cost for policy B as $C_{2}\left(b_{d}, b_{2 u}\right)$. We define the permissible set $B_{1}^{*}$ of $C_{1}\left(b_{1 u}\right)$, and $B_{2}^{*}$ of $C_{2}\left(b_{d}, b_{2 u}\right)$ as the sets of all the optimal burn-in times that minimize $C_{1}\left(b_{1 u}\right)$, and $C_{2}\left(b_{d}, b_{2 u}\right)$; that is,

$$
\begin{aligned}
& \left.B_{1}^{*} \equiv\left\{0 \leq t \leq \infty: C_{1}(t)=\min _{0 \leq s \leq \infty} C_{1}(s)\right)\right\} \\
& \left.B_{2}^{*} \equiv\left\{0 \leq t, t^{\prime} \leq \infty: C_{2}\left(t, t^{\prime}\right)=\min _{0 \leq s, s^{\prime} \leq \infty} C_{2}\left(s, s^{\prime}\right)\right)\right\}
\end{aligned}
$$

and the optimal burn-in $b_{1 u}^{*}$, and $b_{d}^{*}, b_{2 u}^{*}$ as

$$
\begin{aligned}
& b_{1 u}^{*} \equiv \inf B_{1}^{*} \\
& \left(b_{d}^{*}, b_{2 u}\right) \equiv \inf B_{2}^{*}
\end{aligned}
$$




\subsection{Scenario $A-$ Policy $A$ is performed}

Figure 2 shows the process for a one-stage burn-in policy. A burn-in procedure is first carried out within time interval $\left(0, b_{1 u}\right)$ under a normal operating environment, and then the product is installed when a building is constructed.

Considering the above condition leads us to the following result.

Theorem 1 If products are sold with FRW and a one-stage burn-in policy, then the mean total warranty cost for the products is given by

$$
C_{1}\left(b_{1 u}\right)=\left(\nu_{1}\left(b_{1 u}\right)+c_{4}\right)\left(M_{1}(w)+M_{2}(w)+1\right)-c_{4}
$$

where

$$
\begin{aligned}
& \nu_{1}\left(b_{1 u}\right)=c_{0}+c_{1 u}+c_{2 u} \frac{\int_{0}^{b_{1 u}} \bar{F}_{u}(t) d t}{\bar{F}_{u}\left(b_{1 u}\right)}+c_{3} \frac{F_{u}\left(b_{1 u}\right)}{\bar{F}_{u}\left(b_{1 u}\right)}, \\
& M_{1}(w)=F_{1 d}\left(t_{0}\right)\left(1+M_{0}(w)\right), \\
& M_{2}(w)=\left(1-F_{1 d}\left(t_{0}\right)\right) \int_{0}^{w}\left(1+M_{0}(w-t)\right) d F_{1 u}(t), \\
& M_{0}(w)=\sum_{k=1}^{\infty} F_{p 1}^{(k)}(w) .
\end{aligned}
$$

Furthermore, the optimal burn-in time $b_{1 u}^{*}$ must satisfy $b^{*}<t_{1}$.

Proof. See Appendix.

In order to obtain the optimal burn-in time from Eq. (3), we need to approximate the renewal function, $M_{0}(w)$. Here we use an approach from Xie [22] to achieve this goal. Given a renewal function

$$
m(t)=F(t)+\int_{0}^{t} m(t-x) d F(x)
$$

Suppose it is desired to approximate $m(t)$ with $0 \leq t \leq T^{*}$. Partition the time period $\left[0, T^{*}\right]$ into $N$ subintervals: $0=T_{0}<T_{1}<\ldots<T_{L}=T^{*}$. Xie [22] develops the following recursive approximations to $m\left(T_{i}\right)$ :

$$
m\left(T_{i}\right)=\frac{F\left(T_{i}\right)+S_{i}-F\left(T_{i}-T_{i-\frac{1}{2}}\right) m\left(T_{i-1}\right)}{1-F\left(T_{i}-T_{i-\frac{1}{2}}\right)}, 1 \leq i \leq N
$$


with $m\left(T_{0}\right)=0$, where $S_{1}=0$,

$$
S_{i}=\sum_{j=1}^{i-1} F\left(T_{i}-T_{j-\frac{1}{2}}\right)\left(m\left(T_{j}\right)-m\left(T_{j-1}\right)\right), 2 \leq i \leq N
$$

and

$$
T_{i-\frac{1}{2}}=\frac{1}{2}\left(T_{i-1}+T_{i}\right)
$$

To obtain the optimal burn-in time $b_{1 u}$, commonly used heuristic methods such as GA (Genetic Algorithm), SA(Simulated Annealing algorithm), or TS (Tabu search) can be applied for optimizing the burn-in times (see [23]).

\subsection{Scenario $B-$ Policy $B$ is performed}

Figure 3 shows the process for a the two-stage burn-in policy. A burn-in procedure is first carried out within $\left(0, b_{d}\right)$ under the dormant environment, and then another burn-in procedure under the normal operating environment within $\left(b_{d}, b_{d}+b_{2 u}\right)$. The product is installed in a building at time $b_{d}+b_{2 u}$, and commissioned at time $b_{d}+b_{2 u}+t_{0}$.

At stage one in this policy, the mean burn-in cost is

$$
\nu_{2}\left(b_{d}\right)=c_{0}+c_{1 d}+c_{2 d} \frac{\int_{0}^{b_{d}} \bar{F}_{d}(t) d t}{\bar{F}_{d}\left(b_{d}\right)}+c_{3} \frac{F_{d}\left(b_{d}\right)}{\bar{F}_{d}\left(b_{d}\right)}
$$

Denote the life distribution of a product that survives after burn-in time $b_{d}$ by $F_{2}(t)$ within time $\left(b_{d}, b_{d}+b_{2 u}\right)$. At stage two of the two-stage burn-in policy, the mean burn-in cost is

$$
\nu_{3}\left(b_{2 u}\right)=c_{1 u}+c_{2 u} \frac{\int_{0}^{b_{2 u}} \bar{F}_{2}(t) d t}{\bar{F}_{2}\left(b_{2 u}\right)}+c_{3} \frac{F_{2}\left(b_{2 u}\right)}{\bar{F}_{2}\left(b_{2 u}\right)}
$$

The life distribution of the product within time interval $\left(b_{d}+b_{2 u}, b_{d}+b_{2 u}+t_{0}\right)$ is assumed to be $F_{2 d}(t)$ if the product has survived from burn-in time $b_{d}+b_{2 u}$, and the life distribution of the product is assumed to be $F_{2 u}(t)$ if the product is at the normal operating state and does not fail to operate at commissioning time $b_{d}+b_{2 u}+t_{0}$.

If the product fails to operate at time $b_{d}+b_{2 u}+t_{0}$, the expected number of replacements for the product within time interval $\left(b_{d}+b_{2 u}+t_{0}, b_{d}+b_{2 u}+t_{0}+w\right)$ 


$$
M_{4}(w)=F_{2 d}\left(t_{0}\right)\left(1+M_{3}(w)\right)
$$

where $M_{3}(w)=\sum_{k=1}^{\infty} F_{p 2}^{(k)}(w)$, and $F_{p 2}(w)$ is the life distribution of a product after burn-in period $b_{d}+b_{2 u}$ in the two-stage burn-in policy.

If the product fails within time interval $\left(b_{d}+b_{2 u}+t_{0}, b_{d}+b_{2 u}+t_{0}+w\right)$, the expected number of replacements within this time interval is given by

$$
M_{5}(w)=\left(1-F_{2 d}\left(t_{0}\right)\right) \int_{0}^{w}\left(1+M_{3}(w-t)\right) d F_{2 u}(t)
$$

Theorem 2 If products are sold with FRW and a two-stage burn-in policy, then the mean total warranty cost for the products is given by

$$
C_{2}\left(b_{d}, b_{2 u}\right)=\left(\nu_{2}(b)+\nu_{3}(b)+c_{4}\right)\left(M_{4}(w)+M_{5}(w)+1\right)-c_{4}
$$

Furthermore, the optimal burn-in time $b_{d}^{*}, b_{2 u}^{*}$ must satisfy $b_{d}^{*}, b_{2 u}^{*}<t_{1}$.

The proof can be completed by using the one for Theorem 1 in [14] and mimicking the proof of Theorem 2 in this paper.

For both policy A and policy $\mathrm{B}$, the permissible sets of $B_{1}^{*}$, and $B_{2}^{*}$ are subsets of $\left[0, t_{1}\right]$.

\subsection{Discussion}

In real applications, the above two burn-in policies can be selected on the basis of different scenarios.

When the burn-in period $b_{d}=0$, and the fixed setup cost $c_{1 d}=0$, the mean total warranty cost $C_{2}\left(b_{d}, b_{2 u}\right)$ of from Policy B in Theorem 3 is equivalent to $C_{1}\left(b_{1 u}\right)$ from Policy A in Theorem 2.

If the length of stage two in Policy $\mathrm{A}$ is set to the same as that for the burn-in time in Policy B, namely, $b_{2 u}=b_{1 u}$, then $C_{2}\left(b_{d}, b_{2 u}\right)>C_{1}\left(b_{1 u}\right)$. This means the mean total warranty cost incurred in Policy B is larger than that in Policy A.

$C_{2}\left(b_{d}, b_{2 u}\right)$ can be less than $C_{1}\left(b_{1 u}\right)$ if the length of the burn-in period under a normal operating environment in Policy B is shorter than that in Policy A. 


\section{A special case}

The failure rate function in a dormant state is usually lower than a normal operating state. The difference between the two failure rate functions might result from different load conditions and operating environments, hence, the link between them can vary. Here a simple example is given to investigate the policies discussed in the paper.

Assume that the relationship between the failure rates of the product at a dormant state and at a normal operating state is

$$
r_{d}(t)=\alpha r_{u}(\beta t)
$$

where $\alpha, \beta \in(0,1)$, and are called adjustment factors in what follows.

It follows that

$$
F_{d}(t)=1-e^{-\int_{0}^{t} \alpha r_{u}(\beta x) d x}
$$

\subsection{Policy A case}

In Policy A, the cumulative distribution function of a product that survives after burn-in time $b_{1 u}$ is

$$
\begin{aligned}
F_{p 1}(t) & =\frac{F_{u}\left(b_{1 u}+t\right)-F_{u}\left(b_{1 u}\right)}{\bar{F}_{u}\left(b_{1 u}\right)} \\
& =1-e^{-\int_{b_{1 u}}^{b_{1 u}+t} r_{u}(x) d x}
\end{aligned}
$$

Denote $r_{p 1}(t)=\frac{1}{F_{p 1}(t)} \frac{d F_{p 1}(t)}{d t}=r_{u}\left(b_{1 u}+t\right)$. Hence, the life distribution of products survived from the burn-in time $b_{1 u}, F_{1 d}(t)$, is given by

$$
\begin{aligned}
F_{1 d}(t) & =1-e^{-\alpha \int_{0}^{t} r_{p 1}(\beta x) d x} \\
& =1-e^{-\alpha \int_{0}^{t} r_{u}\left(b_{1 u}+\beta x\right) d x}
\end{aligned}
$$

Denote $F_{3}(t)=\frac{F_{1 d}\left(t_{0}+t\right)-F_{1 d}\left(t_{0}\right)}{\bar{F}_{1 d}\left(t_{0}\right)}$ and $r_{3}(t)=\frac{1}{\bar{F}_{3}(t)} \frac{d F_{3}(t)}{d t}=\alpha r_{u}\left(b_{1 u}+\beta t_{0}+\beta t\right)$, then

$$
\begin{aligned}
F_{1 u}(t) & =1-e^{-\frac{1}{\alpha} \int_{0}^{t} r_{3}(x / \beta) d x} \\
& =1-e^{-\int_{0}^{t} r_{u}\left(b_{1 u}+\beta t_{0}+x\right) d x}
\end{aligned}
$$




\subsection{Policy B case}

In the two-stage burn-in policy, the cumulative distribution function of a product that survives after burn-in time $b_{d}$ at a dormant stage for Policy B is

$$
\begin{aligned}
F_{d 0}(t) & =\frac{F_{d}\left(b_{d}+t\right)-F_{d}\left(b_{d}\right)}{\bar{F}_{d}\left(b_{d}\right)} \\
& =1-e^{-\int_{b_{d}}^{b_{d}+t} \alpha r_{u}(\beta x) d x}
\end{aligned}
$$

Denote $r_{d 0}(t)=\frac{1}{F_{d 0}(t)} \frac{d F_{d 0}(t)}{d t}=\alpha r_{u}\left(\beta\left(b_{d}+t\right)\right)$. Hence, $F_{2}(t)$ is given by

$$
\begin{aligned}
F_{2}(t) & =1-e^{-\frac{1}{\alpha} \int_{0}^{t} r_{d 0}(x / \beta) d x} \\
& =1-e^{-\int_{0}^{t} r_{u}\left(\beta b_{d}+x\right) d x}
\end{aligned}
$$

and $F_{p 2}(t)$ is given by

$$
\begin{aligned}
F_{p 2}(t) & =\frac{F_{2}\left(b_{2 u}+t\right)-F_{2}\left(b_{2 u}\right)}{\bar{F}_{2}\left(b_{2 u}\right)} \\
& =1-e^{-\int_{b_{2 u}}^{b_{2 u}+t} r_{u}\left(\beta b_{d}+x\right) d x}
\end{aligned}
$$

Denote $r_{p 2}(t)=\frac{1}{F_{p 2}(t)} \frac{d F_{p 2}(t)}{d t}=r_{u}\left(\beta b_{d}+b_{2 u}+t\right)$. Hence, $F_{2 d}(t)$ is given by

$$
\begin{aligned}
F_{2 d}(t) & =1-e^{-\alpha \int_{0}^{t} r_{p 2}(\beta x) d x} \\
& =1-e^{-\alpha \int_{0}^{t} r_{u}\left(\beta b_{d}+b_{2 u}+\beta x\right) d x}
\end{aligned}
$$

Denote $F_{4}(t)=\frac{F_{2 d}\left(t_{0}+t\right)-F_{2 d}\left(t_{0}\right)}{\bar{F}_{2 d}\left(t_{0}\right)}$ and $r_{4}(t)=\frac{1}{\bar{F}_{4}(t)} \frac{d F_{4}(t)}{d t}=\alpha r_{u}\left(\beta b_{d}+b_{2 u}+\beta\left(t_{0}+\right.\right.$ t)), then

$$
\begin{aligned}
F_{2 u}(t) & =1-e^{-\frac{1}{\alpha} \int_{0}^{t} r_{4}(x / \beta) d x} \\
& =1-e^{-\int_{0}^{t} r_{u}\left(\beta b_{d}+b_{2 u}+\beta t_{0}+x\right) d x}
\end{aligned}
$$

\section{A numerical example}

For burn-in to be effective, lifetimes should have high failure rates initially and then improve. The class of lifetimes having bathtub-shaped failure rates 
has this property. This section consider a distribution introduced by Xie and Lai [24] to investigate the relationship between the total warranty cost and burn-in times.

Xie and Lai [24] introduced the following life distribution.

$$
F_{u}(t)=1-e^{-\left(\lambda_{1} t\right)^{\lambda_{2}}-\left(\lambda_{3} t\right)^{\lambda_{4}}}, t \geq 0
$$

and the failure rate function is

$$
r_{u}(t)=\lambda_{1} \lambda_{2}\left(\lambda_{1} t\right)^{\lambda_{2}-1}+\lambda_{3} \lambda_{4}\left(\lambda_{3} t\right)^{\lambda_{4}-1}, t \geq 0
$$

With different parameters, $r_{u}(t)$ can describe different patterns of bathtub curves. The first change point of this failure rate function is given by

$$
x_{1}=\left(\frac{\left(1-\lambda_{4}\right) \lambda_{4} \lambda_{3}^{\lambda_{4}}}{\left(\lambda_{2}-1\right) \lambda_{2} \lambda_{1}^{\lambda_{2}}}\right)^{\frac{1}{\lambda_{2}-\lambda_{4}}}
$$

Let $c_{0}=100, c_{1 u}=2, c_{2 u}=0.1, c_{3}=10, c_{4}=50, \alpha=0.2, \beta=0.4, t_{0}=0.3$, $w=0.5, c_{1 d}=1$, and $c_{2 d}=0.05$. The mean total warranty cost are shown in Table 1 for four groups of parameters $\lambda_{1}, \lambda_{2}, \lambda_{3}$, and $\lambda_{4}$.

As Policy A might find more applications from the real world, we pay more attention to Policy A than Policy B in the following.

Table 1 shows that all of optimal values of $b_{1 u}$ are less than $x_{1}$, which verifies the results in Theorem 1 and 2. Compare the costs based on Policy A with Policy B, only the third group of the parameters makes the cost of Policy B (116.60) less than that of Policy A (118.91), which indicates that the two policies can find their application in different settings.

For policy A, the change of the mean total warranty costs based on Eq. (3) vs. the values of $b_{1 u}$ is shown in Figure 4 and 5. In the four figures, the X-axis represents $b_{1 u}$ values and the $\mathrm{Y}$-axis is the mean total warranty costs. The costs increase rapidly after the first change point.

When setting $\alpha_{1}=0.2, \alpha_{2}=2, \alpha_{3}=5$, and $\alpha_{4}=0.5$, we have the following results. Figure 6 shows the change of optimal burn-in time with $\alpha$ values when the values of $\beta$ are set to $0.1,0.5$, and 1 , respectively. It shows that the optimal burn-in times increase rapidly when $\alpha$ change from 0.1 to 1 , and $\beta=1$, whereas the optimal burn-in times increase slowly when $\beta=0.1$. Figures 7 shows the change of the optimal mean total warranty costs when the values of $\alpha$ changes. It shows that in all cases the optimal mean total warranty costs increase. This 
is true as the bigger the value of $\alpha$ is, the bigger the failure rate is, and hence burn-in costs and time increase.

\section{Conclusions}

This paper considers two policies that can be used to two different scenarios. The first policy performs a burn-in procedure for products under normal operating environment. The products are then installed, but not used when a building is constructed. After a time period, the building is commissioned and the products are used. The second policy first conducts a burn-in procedure for products in a dormant mode for a time period, and then in a normal operating environment for a time period. After the two burn-in stages, the products are then installed when a building is constructed, and put into use when the building is commissioned. Costs for the two burn-in policies have been derived, and the relationships between the life distributions at different stages are discussed. Numerical examples are given to verify the results of the mathematical models.

\section{Acknowledgments}

The authors would like to thank Professor Min Xie, Professor Zhizhong Li, and two anonymous reviewers for their comments and suggestions, which have resulted in a number of improvements in the paper. The authors also wish to thank EPSRC as part of the Innovative Manufacturing Research Centres (IMRC) initiative for their financial support and our industrial partners (EC Harris, Dytecna Limited, EMCOR Rail, INBIS and Quorum Logistics Support).

\section{References}

[1] H. Ward, A. H. Christer, Modelling the re-design decision for a warranted product, Reliability Engineering and System Safety 88 (2) (2005) 181-189.

[2] W. Y. Yun, Y. W. Lee, L. Ferreira, Optimal burn-in time under cumulative free replacement warranty, Reliability Engineering and System Safety 78 (2) (2002) 93-100.

[3] C. S. Kim, I. Djamaludin, D. N. P. Murthy, Warranty and discrete preventive maintenance, Reliability Engineering and System Safety 84 (3) (2004) 301-309. 
[4] B. Rai, N. Singh, A modeling framework for assessing the impact of new time/mileage warranty limits on the number and cost of automotive warranty claims, Reliability Engineering and System Safety 88 (2) (2005) 157-169.

[5] L. Attardi, M. Guida, G. Pulcini, A mixed-weibull regression model for the analysis of automotive warranty data, Reliability Engineering and System Safety 87 (2) (2005) 265-273.

[6] M. Kimura, T. Toyota, S. Yamada, Economic analysis of software release problems with warranty cost and reliability requirement, Reliability Engineering and System Safety 66 (1) (1999) 49-55.

[7] W. Kuo, W. T. K. Chien, T. Kim, Reliability, yield, and stress burn-in, Kluwer Academic Publishers, 1998.

[8] K. O. Kim, W. Kuo, A general model of heterogeneous system lifetimes and conditions for system burn-in, Naval Research Logistics 50 (2003) 364-380.

[9] K. O. Kim, W. Kuo, Some consideration on system burn-in, IEEE Transactions on Reliability 54 (2) (2005) 207-214.

[10] Y. I. Kwon, J. B. Keats, Bayesian burn-in procedures for limited failure populations, International Journal of Production Research 40 (2002) 2547-2555.

[11] S. T. Tseng, J. Tang, I. H. Ku, Determination of burn-in parameters and residual life for highly reliable products, Naval Research Logistics 50 (2002) $1-14$.

[12] J. Mi, Maximization of a survival probability and its application, Journal of Applied Probability 31 (4) (1994) 1026-1033.

[13] N. Ebrahimi, Burn-in and covariates, Journal of Applied Probability 41 (3) (2004) $735-745$.

[14] J. Mi, Warranty policies and burn-in, Naval Research Logistics 44 (1997) 199209.

[15] J. H. Cha, On a better burn-in procedure, Journal of Applied Probability 37 (2000) 1099-1103.

[16] C. L. Wu, C. T. Su, Determination of the optimal burn-in time and cost using an environmental stress approach: a case study in switch mode rectifier, Reliability Engineering and System Safety 76 (1) (2002) 53-61.

[17] J. H. Cha, On optimal burn-in procedures - a generalized model, IEEE Transactions on Reliability 54 (2005) 198-205.

[18] W. R. Blischke, D. N. P. Murthy, Warranty cost analysis, Marcel Dekker, New York, 1994.

[19] H. W. Block, T. H. Savits, Burn-in, Statistical Science 12 (1997) 1-19.

[20] F. Jensen, N. E. Petersen, Burn-in, John Wiley, New York, 1982. 
[21] W. Kuo, Y. Kuo, Facing the headaches of early failures: a state-of-the-art review of burn-in decisions, in: Proc. IEEE, Vol. 71, 1983, pp. 1257-1266,.

[22] M. Xie, On the solution of renewal-type integral equations, Communications in Statistics Simulation and Computation 18 (1) (1989) 281-293.

[23] S. Wu, L. Y. Chan, Performance utility analysis of multi-state systems, IEEE Transactions on Reliability 52 (1) (2003) 14-21.

[24] M. Xie, C. D. Lai, Reliability analysis using an additive Weibull model with bathtub-shaped failure rate function, Reliability Engineering and System Safety 52 (1) (1996) 87-93.

\section{Appendix}

\section{Proof of Theorem 1}

The mean burn-in cost within time interval $\left(0, b_{1 u}\right)$ is obtained by

$$
\nu_{1}\left(b_{1 u}\right)=c_{0}+c_{1 u}+c_{2 u} \frac{\int_{0}^{b_{1 u}} \bar{F}_{u}(t) d t}{\bar{F}_{u}\left(b_{1 u}\right)}+c_{3} \frac{F_{u}\left(b_{1 u}\right)}{\bar{F}_{u}\left(b_{1 u}\right)}
$$

The proof for the above equation can be found in [14].

The product is at the dormant state within time interval $\left(b_{1 u}, b_{1 u}+t_{0}\right)$. At time $b_{1 u}+t_{0}$, if the product fails to operate, a new identical one will replace it. Within time interval $\left(b_{1 u}+t_{0}, b_{1 u}+t_{0}+w\right)$, replacement is made upon failures.

The life distribution of the product within time interval $\left(b_{1 u}, b_{1 u}+t_{0}\right)$ is assumed to be $F_{1 d}(t)$ if the product has survived from burn-in time $b_{1 u}$. If the product does not fail to operate at the commissioning time $b_{1 u}+t_{0}$, the life distribution of the product is assumed to be $F_{1 u}(t)$ after the dormant state.

If the product fails to operate at commissioning time $b_{1 u}+t_{0}$, the expected number of replacements within time interval $\left(b_{1 u}+t_{0}, b_{1 u}+t_{0}+w\right)$ is

$$
M_{1}(w)=F_{1 d}\left(t_{0}\right)\left(1+M_{0}(w)\right)
$$

where $M_{0}(w)=\sum_{k=1}^{\infty} F_{p 1}^{(k)}(w)$, and $F_{p 1}(w)$ is the life distribution of a product after burn-in period $b_{1 u}$ in the one-stage burn-in policy.

If the first failure of the product is in time interval $\left(b_{1 u}+t_{0}, b_{1 u}+t_{0}+w\right)$, the expected number of replacements within this time interval is

$$
M_{2}(w)=\left(1-F_{1 d}\left(t_{0}\right)\right) \int_{0}^{w}\left(1+M_{0}(w-t)\right) d F_{1 u}(t)
$$


Using the proof for Theorem 1 in [14], we can obtain Eq. (3).

It is evident that $\nu_{1}\left(b_{1 u}\right)$ given in Eq. (24) is strictly increasing in $b_{1 u} \geq 0$ (see $[14])$.

Denote $M_{0}^{(i)}(w)=\left.M_{0}(w)\right|_{b_{1 u}=b_{i}}, F_{1 u}^{(i)}(t)=\left.F_{1 u}(i)\right|_{b_{1 u}=b_{i}}$, and $F_{1 d}^{(i)}(t)=\left.F_{1 d}(i)\right|_{b_{1 u}=b_{i}}$, where $i=1,2$. From Theorem 4 in Mi [12] for any given $w \geq 0$ there exists $b_{1} \in\left[0, t_{1}\right]$, such as $M_{0}^{(1)}(w) \geq M_{0}^{(2)}(w), \forall b_{2}>t_{1}$. As the failure rate function is assumed to be increasing after the first change point $x_{1}, F_{1 d}^{(1)}(w) \geq F_{1 d}^{(2)}(w)$, $F_{1 u}^{(1)}(w) \geq F_{1 u}^{(2)}(w)$, and

$\int_{0}^{w}\left(1+M_{0}^{(2)}(w-t)\right) d F_{1 u}^{(2)}(t) \geq \int_{0}^{w}\left(1+M_{0}^{(1)}(w-t)\right) d F_{1 u}^{(1)}(t)$.

Let

$$
\begin{aligned}
& F_{1 d}^{(2)}(w)=F_{1 d}^{(1)}\left(b_{1}, w\right)+\Delta_{1}=f_{1 d}+\Delta_{1}, \\
& 1+M_{0}^{(2)}(w)=1+M_{0}^{(1)}\left(b_{1}, w\right)+\Delta_{2}=m_{0}+\Delta_{2},
\end{aligned}
$$

and

$$
\begin{aligned}
\int_{0}^{w}\left(1+M_{0}^{(2)}(w-t)\right) d F_{1 u}^{(2)}(t) & =\int_{0}^{w}\left(1+M_{0}^{(1)}(w-t)\right) d F_{1 u}^{(1)}(t)+\Delta_{3} \\
& =m_{1}+\Delta_{3}
\end{aligned}
$$

where $\Delta_{1}, \Delta_{2}, \Delta_{3}>0$. As $1>f_{1 d}+\Delta_{1}$ and $m_{0}>m_{1}$, hence

$$
\begin{aligned}
& M_{1}^{(2)}(w)+M_{2}^{(2)}(w)+1-M_{1}^{(1)}(w)-M_{2}^{(1)}(w)-1 \\
= & \left(f_{1 d}+\Delta_{1}\right)\left(m_{0}+\Delta_{2}\right)+\left(1-f_{1 d}-\Delta_{1}\right)\left(m_{1}+\Delta_{3}\right)-f_{1 d} m_{0}-\left(1-f_{1 d}\right) m_{1} \\
= & f_{1 d} \Delta_{2}+m_{0} \Delta_{1}+\Delta_{1} \Delta_{2}+\Delta_{3}-m_{1} \Delta_{1}-f_{1 d} \Delta_{3}-\Delta_{1} \Delta_{3} \\
\geq & f_{1 d} \Delta_{2}+\Delta_{1} \Delta_{2} \\
\geq & 0
\end{aligned}
$$

Therefore, $M_{1}(w)+M_{2}(w)+1$ is increasing in $b_{1 u}$ if $b_{1 u}>t_{1}$. This proves that the optimal burn-in time $b_{1 u}^{*}$ must satisfy $b_{1 u}^{*}<t_{1}$. 
Table 1

Different costs incurred

\begin{tabular}{c|cccc|c|cc|ccc}
\hline & \multicolumn{3}{|c|}{ Parameters } & Change point & \multicolumn{2}{c|}{ Policy A } & \multicolumn{3}{c}{ Policy B } \\
\cline { 2 - 11 } Group & $\lambda_{1}$ & $\lambda_{2}$ & $\lambda_{3}$ & $\lambda_{4}$ & $x_{1}$ & $b_{1 u}$ & $C_{1}\left(b_{1 u}\right)$ & $b_{d}$ & $b_{2 u}$ & $C_{2}\left(b_{d}, b_{2 u}\right)$ \\
\hline 1 & 0.1 & 1.5 & 1 & 0.1 & 2.59 & 0.76 & 131.79 & 0.1 & 1.1 & 145.91 \\
2 & 0.8 & 1.1 & 0.06 & 0.3 & 1.06 & 0.15 & 192.12 & 0.1 & 0.2 & 270.74 \\
3 & 0.5 & 10 & 0.0001 & 0.1 & 0.9133 & 0.48 & 118.91 & 0.05 & 0.5 & 116.60 \\
4 & 0.2 & 2 & 5 & 0.5 & 3.66 & 0.33 & 273.72 & 0.15 & 0.4 & 405.11 \\
\hline
\end{tabular}

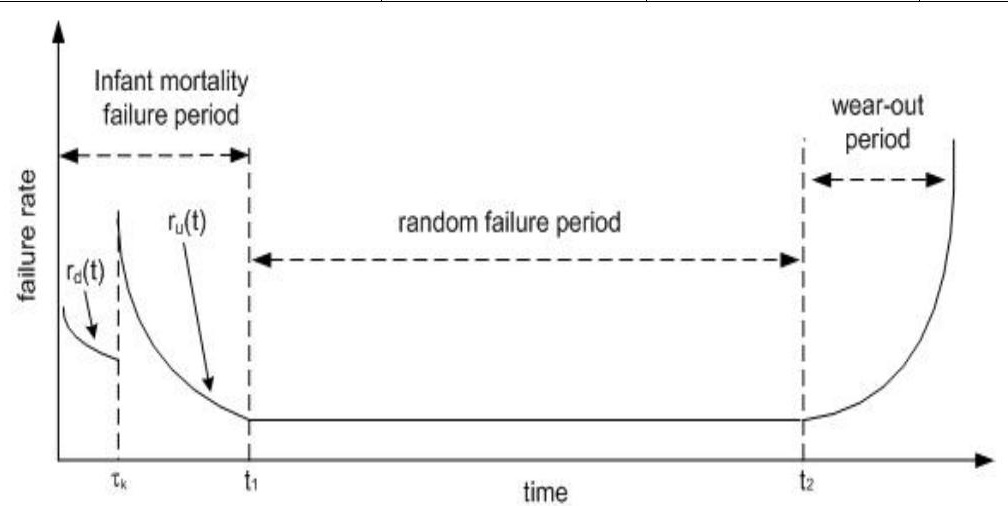

Fig. 1. A broken bathtub curve.

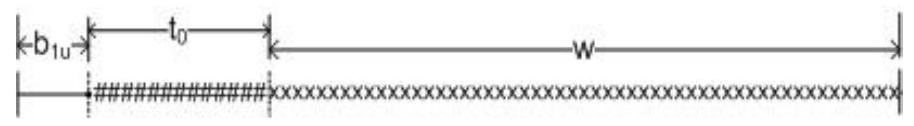

— : burn in \#\#\#: dormant xxx: operating

Fig. 2. one-stage burn-in policy.

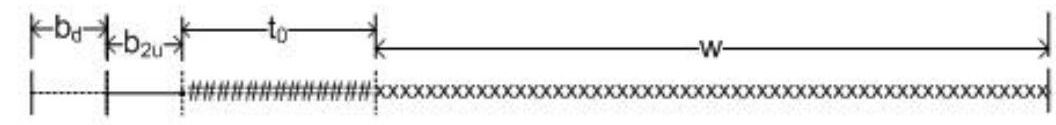

......: burn in stage one - burn in stage two \#\#. dormant xxx: operating

Fig. 3. two-stage burn-in policy. 

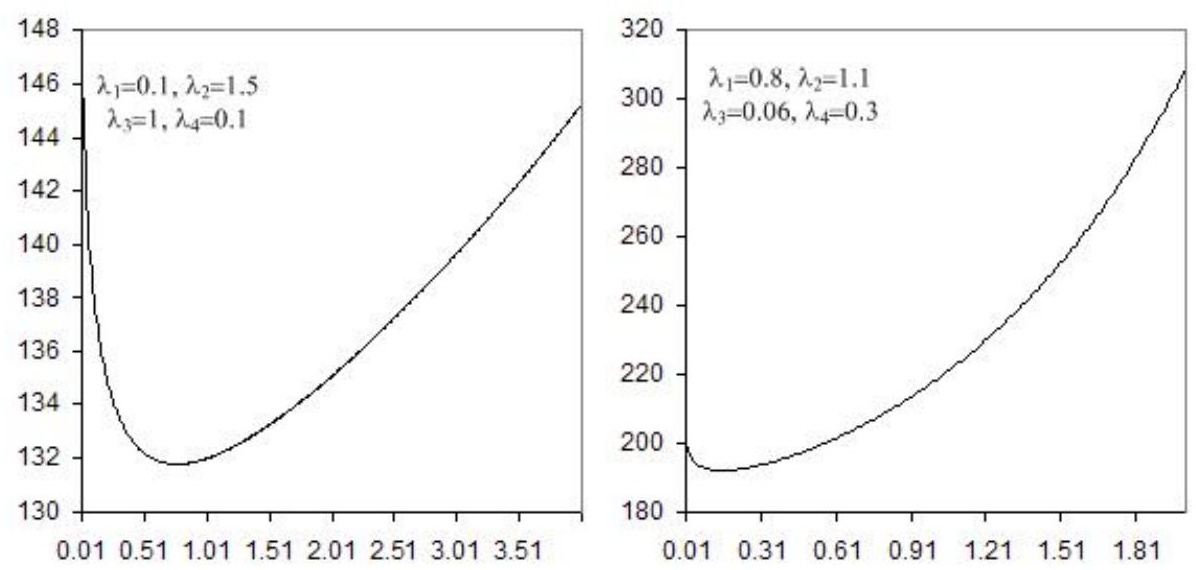

Fig. 4. Expected cost vs. burn-in time for two different life distributions as shown.
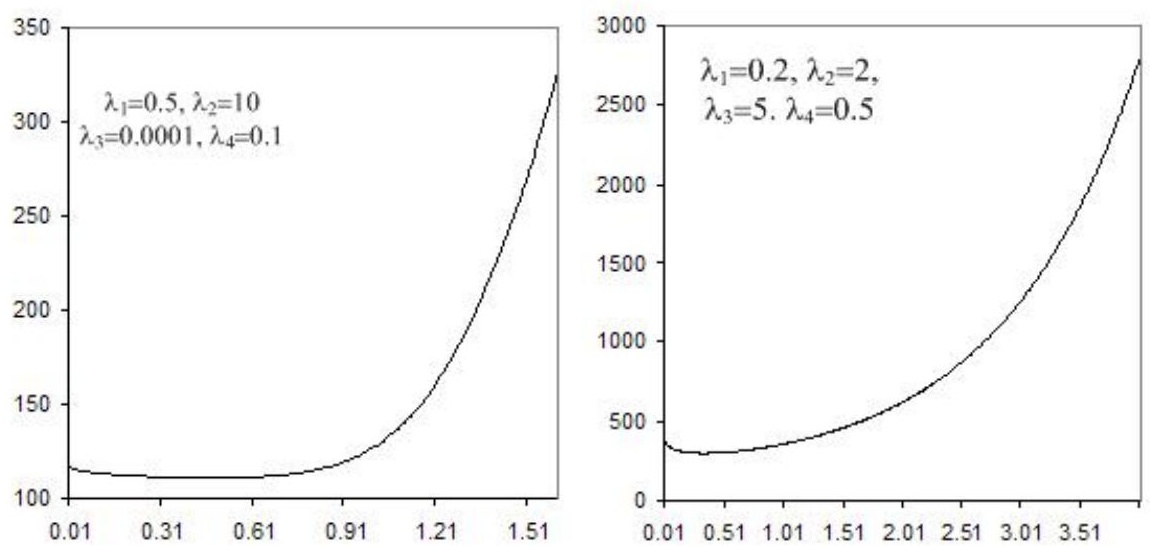

Fig. 5. Expected cost vs. burn-in time for two different life distributions as shown.

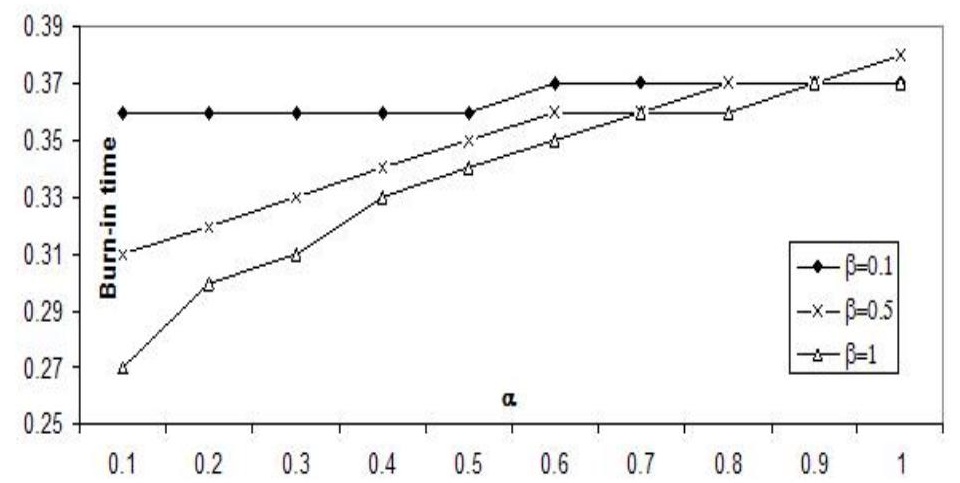

Fig. 6. Optimal burn-in times when the values of $\alpha$ change from 0.1 to 1 , and $\beta=0.1$, 0.5 , and 1 . 


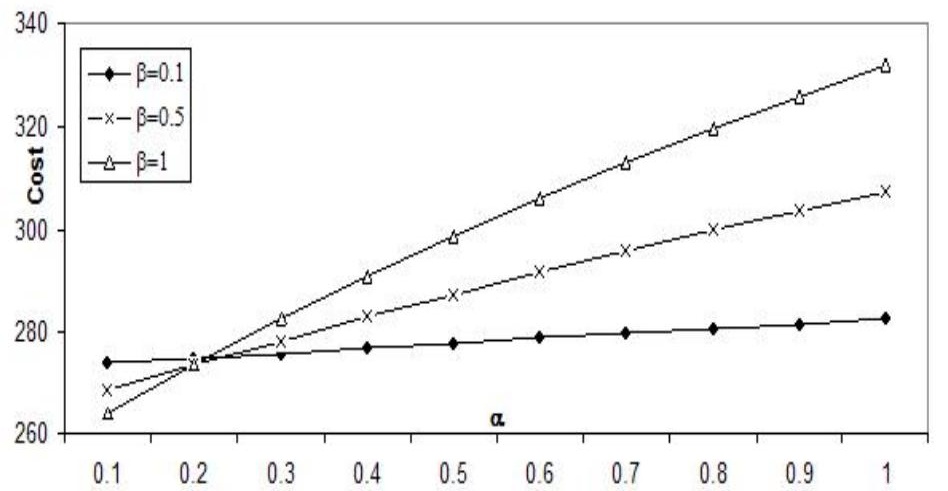

Fig. 7. Costs when the values of $\alpha$ change from 0.1 to 1 , and $\beta=0.1,0.5$, and 1 . 


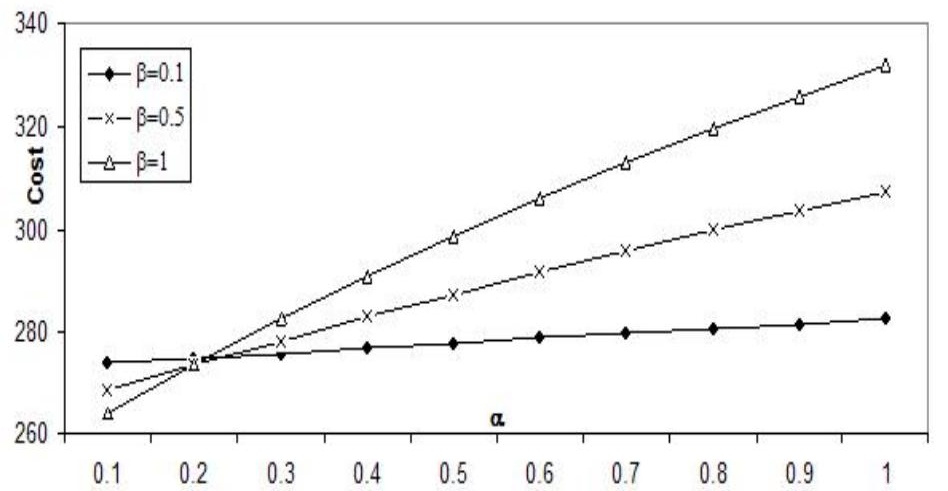

Fig. 7. Costs when the values of $\alpha$ change from 0.1 to 1 , and $\beta=0.1,0.5$, and 1 . 